\title{
PUBLICACIÓN DE LOS PROYECTOS DE INVESTIGACIÓN PARA LA TITULACIÓN COMO MÉDICOS ESPECIALISTAS DE UNA UNIVERSIDAD PERUANA, 2007-2010
}

\author{
Ray Ticse ${ }^{1,2, a}$, Patricia Ygreda ${ }^{2, b}$, Frine Samalvides ${ }^{1,2, c}$
}

\begin{abstract}
RESUMEN
Con el objetivo de determinar la frecuencia de publicación en una revista científica, de los proyectos de investigación realizados para la obtención del título de médico especialista por médicos residentes egresados de la Facultad de Medicina de la Universidad Peruana Cayetano Heredia, se realizó una búsqueda en Google académico, Pubmed, bases de datos biomédicas y revistas de sociedades científicas medicas peruanas. De 351 médicos residentes egresados entre los años 2007-2010, 199 (65,5\%) culminaron su proyecto de investigación y 47 (23,6\%) lo publicaron en una revista científica. Según la especialidad el área de medicina tuvo la mayor frecuencia de publicaciones. Todas las publicaciones se realizaron en revistas en español, siendo la mayoría en revistas indizadas en bases de datos regionales. Concluimos que el $23,6 \%$ de los proyectos de investigación realizados para la titulación como médicos especialistas logran ser publicados, la mayoría de veces en revistas científicas de baja visibilidad.
\end{abstract}

Palabras clave: Residencia médica; Publicaciones científicas y técnicas; Proyectos de investigación (fuente: DeCS BIREME).

\section{PUBLICATION OF RESEARCH PROJECTS FOR CERTIFICATION AS MEDICAL SPECIALISTS AT A PERUVIAN UNIVERSITY, 2007-2010}

\begin{abstract}
In order to determine the frequency of publication in a scientific journal of the research projects done for medical specialty certification, a search was conducted in Google Scholar, Pubmed, biomedical databases and Peruvian medical society journals. These publications were research projects carried out by medical residents graduated from the Faculty of Medicine at the Universidad Peruana Cayetano Heredia, to obtain the certification of medical specialist. Of 351 medical residents graduated between the years 2007-2010, 199 (65.5\%) completed their research project and $47(23.6 \%)$ published it in a scientific journal. The "medicine" (non-surgical) specialty area had the highest frequency of publications. All publications were in Spanish journals, the majority in indexed journals in regional databases. We conclude that $23.6 \%$ of the research projects for certification as medical specialists are published, most often in low visibility journals.
\end{abstract}

Key words: Residency, medical; Scientific and technical publications; Research design (source: MeSH NLM).

\section{INTRODUCCIÓN}

La publicación científica es el principal medio para difundir el conocimiento obtenido de una investigación. En Latinoamérica, los países con mayores publicaciones son Brasil y Argentina. En cambio, en el Perú es muy reducida, con aproximadamente el $0,002 \%$ de lo alcanzado por Brasil ${ }^{(1)}$. Es importante mencionar que algunos países han incrementado su número de publicaciones, no necesariamente debido a un mayor número de proyectos realizados, sino más bien a un mayor interés por publicar ${ }^{(2)}$.

En el Perú, el proceso de selección de postulantes al residentado médico (RM) se realiza mediante un concurso diseñado por la Asociación Peruana de Facultades de Medicina (ASPEFAM). Las vacantes a las especialidades se clasifican en: clínicas, quirúrgicas y

Hospital Nacional Cayetano Heredia. Lima, Perú.

Facultad de Medicina, Universidad Peruana Cayetano Heredia. Lima, Perú

Médico internista endocrinólogo; magíster en Epidemiología Clínica; ${ }^{\mathrm{b}}$ médico cirujano; ${ }^{\mathrm{c}}$ médico infectólogo

* El informe preliminar de este estudio fue presentado en el IV Congreso Peruano e Internacional de Educación Médica de la Asociación Peruana de Facultades de Medicina en abril de 2013.

Recibido: 01-09-13 Aprobado: 12-03-14

Citar como: Ticse R, Ygreda P, Samalvides F. Publicación de los proyectos de investigación para la titulación como médicos especialistas de una universidad peruana, 2007-2010. Rev Peru Med Exp Salud Publica. 2014;31(2):292-6. 
subespecialidades. El Comité Nacional de Residentado Médico (CONAREME) es el responsable de garantizar la admisión y formación médica en todos los programas de RM del país ${ }^{(3,4)}$. Desde el 2006, con el propósito de generar investigación en el RM, el CONAREME solicita la presentación de un trabajo de investigación culminado para acceder al título de especialista ${ }^{(5)}$.

Es importante que los egresados del RM tengan competencias en investigación, porque les permite ser capaces de plantear soluciones a los problemas de salud y generar conocimiento en sus especialidades. Estas competencias deben ser impartidas durante el $\mathrm{RM}$, debido a que este tiene propósitos académicos y profesionales ${ }^{(5)}$. La publicación de los resultados de la investigación científica, realizada en revistas indizadas nacionales o internacionales, permite a las universidades posicionarse dentro de la comunidad científica, fortalecer sus redes de colaboración y obtener prestigio académico. Por este motivo es importante que las instituciones educativas realicen periódicamente mediciones sobre la producción científica de sus diversos programas ${ }^{(6)}$. El objetivo de este estudio fue determinar la frecuencia de publicación de los proyectos de investigación realizados por los egresados del programa de RM en una universidad de Lima, Perú.

\section{EL ESTUDIO}

Estudio transversal, donde la población estuvo formada por los proyectos de investigación culminados, realizados para la obtención del título de médico especialista por los egresados del programa de RM de la Facultad de Medicina de la Universidad Peruana Cayetano Heredia, durante el periodo 2007-2010. La información de los médicos residentes fue brindada por la Unidad de Investigación, Ciencia y Tecnología (UICT) de la Facultad de Medicina. Se recolectó la siguiente información: nombres de los autores, sede donde realizaron el RM, título del proyecto de investigación, resumen del proyecto de investigación y palabras clave.

En cuanto a los proyectos, se consideró como "proyecto presentado" a todo proyecto de investigación que hubiese sido presentado por el alumno a la UICT de la facultad de medicina, para su evaluación y aprobación. Se consideró como "proyecto aprobado" a todo aquel proyecto que hubiese sido aprobado por la UICT (se requiere de esta aprobación y de la aprobación del comité de ética institucional antes del inicio de la ejecución del proyecto). Se denominó "proyecto culminado" a todo aquel cuyo informe final tuviese nota mínima aprobatoria (calificación $\geq 13$ puntos sobre 20 posibles) de parte de un jurado calificador, el cual está conformado por tres profesores designados por la UICT.
Se realizó una búsqueda en Google Scholar, SciELO (Scientific Electronic Library Online), LIPECS (Literatura Peruana en Ciencias de la Salud), LILACS (Literatura Latinoamericana y del Caribe en Ciencias de la Salud) y Pubmed (National Library of Medicine) siguiendo estrategias de búsqueda previamente descritas para este propósito ${ }^{(7-9)}$. De manera complementaria, se revisaron la base de datos Scopus y las páginas web de las sociedades científicas médicas nacionales registradas ante el Colegio Médico del Perú. La búsqueda se realizó entre los días 21 y 24 de febrero de 2013.

Para corroborar que la publicación correspondía al médico residente, se revisó el artículo, sus palabras clave, así como los datos de filiación del autor: sede docente, especialidad y lugar de procedencia.

Los datos obtenidos de la búsqueda fueron recolectados en una base de datos electrónica en Microsoft Office Excel 2010, a partir de la cual se determinó la frecuencia de las características de los trabajos de investigación publicados por especialidad.

El protocolo de este estudio fue aprobado por el Comité institucional de Ética de la Universidad Peruana Cayetano Heredia (código SIDISI 0000061102, año 2013).

\section{RESULTADOS}

Del total de 351 médicos residentes egresados entre los años 2007-2010; 304 (86,6\%) presentaron un proyecto de investigación, de los cuales $199(65,5 \%)$ fueron culminados, mientras que 47 (23,6\%) fueron publicados. El porcentaje de publicación varió en los diferentes años con $13,1 \%$ para el $2007 ; 29,8 \%$ para el 2008; 27,9\% para el 2009, y 26,3\% para el 2010. De los 47 proyectos publicados, $46(97,9 \%)$ tuvieron como primer autor y como autor corresponsal al médico residente.

Las especialidades quirúrgicas tuvieron el mayor número de trabajos culminados: 63 (31,7\%). El área con mayor porcentaje de publicación fue medicina con $45 \%$, dentro del cual se observó que la especialidad con mayor publicación fue medicina interna $(22,2 \%)$ (Tabla 1).

Del total de proyectos publicados, todos los artículos fueron publicados en español; 45 (93,7\%) proyectos fueron hallados en revistas nacionales. La revista con mayor cantidad de artículos publicados fue la Revista Médica Herediana $(27,7 \%)$, seguida por la Revista de Gastroenterología del Perú (12,8\%); dos 
Tabla 1. Proyectos de investigación según especialidad del médico residente de la Universidad Peruana Cayetano Heredia, 2007-2010

\begin{tabular}{|c|c|c|c|c|}
\hline & $\begin{array}{l}\text { Proyectos } \\
\text { culminados }\end{array}$ & $\begin{array}{l}\text { Proyectos } \\
\text { publicados }\end{array}$ & $\begin{array}{c}\text { Publicados } \\
\text { en revistas } \\
\text { Medline }\end{array}$ & $\begin{array}{l}\text { Relación } \\
\text { entre } \\
\text { proyectos } \\
\text { publicados y } \\
\text { culminados }\end{array}$ \\
\hline & N (\%) & N (\%) & N (\%) & (\%) \\
\hline Cirugía* & $63(31,7)$ & $9(19,1)$ & $2(22,2)$ & $(14,3)$ \\
\hline $\begin{array}{l}\text { Ginecología y } \\
\text { obstetricia }\end{array}$ & $31(15,8)$ & $6(12,8)$ & 0 & $(19,3)$ \\
\hline Medicina* & $60(30,1)$ & $27(57,5)$ & $5(18,5)$ & $(45,0)$ \\
\hline Pediatría & $14(7,1)$ & $2(4,3)$ & 0 & $(14,3)$ \\
\hline Salud mental $^{\dagger}$ & $5(2,5)$ & $0(0)$ & 0 & $(0,0)$ \\
\hline Otros $^{\ddagger}$ & $26(13,1)$ & $3(6,4)$ & 0 & $(11,5)$ \\
\hline \multicolumn{5}{|c|}{ 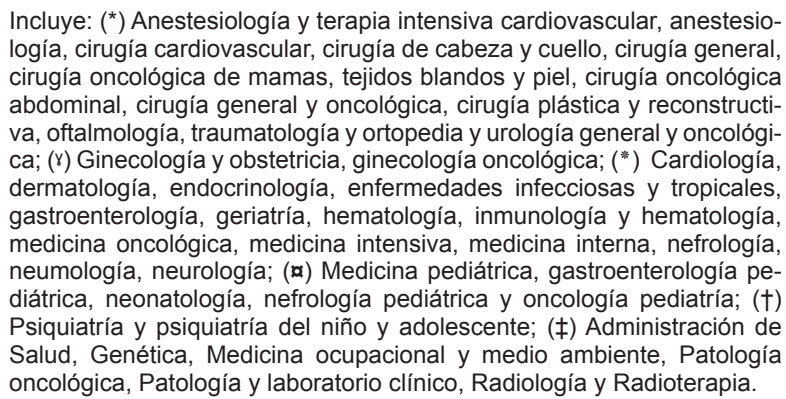 } \\
\hline
\end{tabular}

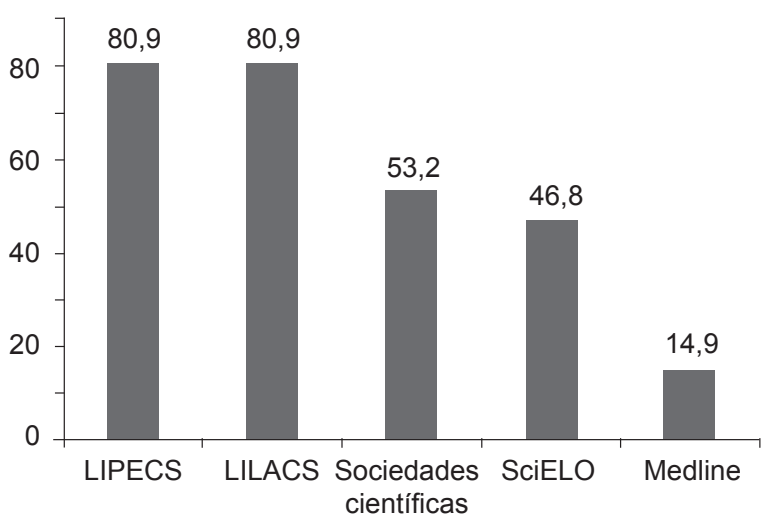

LIPECS: Literatura Peruana en Ciencias de la Salud, LILACS: Literatura Latinoamericana y del Caribe en Ciencias de la Salud, SciELO: Scientific Electronic Library Online

Figura 1. Publicaciones de trabajos de investigación para la titulación como médicos especialistas egresados de la Facultad de Medicina de la Universidad Peruana Cayetano Heredia, 2007-2010

artículos $(4,2 \%)$ fueron publicados en revistas con sede editorial en Chile y $1(2,1 \%)$ en España (Tabla 2$)$.

La mayoría de las publicaciones fueron halladas en las bases de datos de LIPECS y LILACS $(81,3 \%$ cada uno), seguidos por SciELO (48\%) y Medline $(16,7 \%)$ (Figura 1).

Tabla 2. Revistas donde se publicaron los proyectos de investigación para la titulación como médicos especialistas de la Universidad Peruana Cayetano Heredia, 2007-2010

\begin{tabular}{|c|c|c|c|c|c|}
\hline \multirow{2}{*}{ Revista } & \multirow{2}{*}{$\begin{array}{c}\text { Publicaciones } \\
\text { N (\%) }\end{array}$} & \multicolumn{4}{|c|}{ Indización } \\
\hline & & Medline & SciELO & LILACS & LIPECS \\
\hline Revista Médica Herediana & $13(27,7)$ & No & Sí & Sí & Sí \\
\hline Revista de Gastroenterología del Perú & $6(12,8)$ & Sí & Sí & Sí & Sí \\
\hline Acta Cancerológica & $5(10,6)$ & No & No & Sí & Sí \\
\hline Revista de la Sociedad Peruana de Medicina Interna & $5(10,6)$ & No & No & Sí & Sí \\
\hline Actas Peruanas de Anestesiología & $2(4,3)$ & No & No & Sí & Sí \\
\hline Revista Peruana de Ginecología y Obstetricia & $2(4,3)$ & No & No & No & Sí \\
\hline Acta Médica Peruana & $2(4,3)$ & No & Sí & Sí & Sí \\
\hline Archivos Chilenos de Oftalmología & $1(2,1)$ & No & No & Sí & No \\
\hline Revista Peruana de Oftalmología & $1(2,1)$ & No & No & No & No \\
\hline Carcinos & $1(2,1)$ & No & No & No & No \\
\hline Archivos Españoles de Urología & $1(2,1)$ & Sí & Sí & No & No \\
\hline Revista Peruana de Cardiología & $1(2,1)$ & No & No & Sí & Sí \\
\hline Enfermedades del Tórax (Lima) & $1(2,1)$ & No & No & Sí & Sí \\
\hline Revista Chilena de Infectología & $1(2,1)$ & Sí & No & No & No \\
\hline Revista Peruana de Urología & $1(2,1)$ & No & No & No & No \\
\hline Folia Dermatológica & $1(2,1)$ & No & No & Sí & Sí \\
\hline Revista Peruana de Obstetricia y Enfermería & $1(2,1)$ & No & No & No & No \\
\hline Diagnóstico & $1(2,1)$ & No & No & Sí & Sí \\
\hline Revista Peruana de Reumatología & $1(2,1)$ & No & No & No & No \\
\hline
\end{tabular}

SciELO: Scientific Electronic Library Online, LILACS: Literatura Latinoamericana y del Caribe en Ciencias de la Salud, LIPECS: Literatura Peruana en Ciencias de la Salud 


\section{DISCUSIÓN}

Menos de la cuarta parte de los proyectos de investigación culminados por egresados del RM de la Facultad de Medicina de la Universidad Peruana Cayetano Heredia logró ser publicado en una revista científica, en la totalidad de los casos en idioma español. Se observa un incremento a partir del 2008, esto podría deberse a que desde un año antes la Facultad de Medicina exige la presentación del informe final del proyecto culminado en formato de artículo para su publicación en una revista científica.

La publicación de los resultados de la investigación permite a las universidades posicionarse dentro de la comunidad científica, fortalecer sus redes de colaboración y obtener prestigio académico. Generalmente, el tiempo destinado por el programa de RM a estos propósitos es escaso o nulo debido a que su prioridad es el desarrollo de competencias asistenciales. Sin embargo, el RM, por su naturaleza académica y formativa, debe estimular al médico residente culminar y publicar sus proyectos de investigación ${ }^{(5)}$. Esto mejoraría la baja cultura de publicación descrita en nuestro país.

Sobre la cultura de publicación, algunos países han incrementado su producción científica no como consecuencia de culminar más proyectos, sino por estimular la publicación de sus resultados ${ }^{(2)}$. Se han identificado diversos inconvenientes que tienen los médicos residentes para publicar, como: el pensar que sus trabajos no son lo suficientemente importantes para ser publicados, por lo que ni siquiera lo intentan (10); la falta de valoración personal del proyecto realizado; la ausencia de cultura de publicación; la falta de capacitación; el desconocimiento de oportunidades; la calidad de la investigación, y la falta de apoyo docente y de incentivos (11). Si se logra culminar el proyecto, también se tiene la dificultad de no contar con el tiempo suficiente para resumirlo y adaptarlo a las exigencias requeridas para su publicación en revistas científicas ${ }^{(12)}$.

Adaptar la presentación del proyecto culminado al formato de publicación de una revista científica facilita su posterior publicación ${ }^{(12)}$. Desde el 2006, la Facultad de Medicina de la Universidad Peruana Cayetano Heredia exige que la presentación del informe final del proyecto culminado se realice según las normas de publicación de la Revista Médica Herediana (RMH), revista regida por esta facultad. Esto puede explicar dos resultados: primero, que sea la $\mathrm{RMH}$ la revista en donde los egresados lograron mayor número de publicaciones $\mathrm{y}$, segundo, el incremento de la frecuencia de publicaciones observada en el 2008. Sin embargo, luego de este año, dicha frecuencia presentó un leve descenso, por lo que es necesario implementar otras estrategias.
Con el propósito de incrementar la publicación de los proyectos culminados, se pueden evaluar estrategias utilizadas en otros programas de RM. Por ejemplo, una iniciativa que ha aumentado el porcentaje de publicaciones en Norteamérica es el "Annual Research Day", en el cual el personal médico se reúne para presentar los proyectos de investigación que se han elaborado en el transcurso de ese año ${ }^{(13)}$. Algunas facultades de medicina otorgan créditos o puntaje a los profesionales de salud que han publicado trabajos en revistas científicas indizadas a alguna base de datos electrónica de relevancia, cuando postulen a una plaza en alguna institución, mejorando el porcentaje de trabajos enviados a revistas reconocidas $(6,14,15)$.

Ninguna publicación fue lograda durante el RM para esta universidad peruana, esto se podría explicar porque existe una muy baja frecuencia de culminación de proyectos: menos del $2 \%$ durante el RM y menos del $24 \%$ al año de egresado ${ }^{(16)}$. Asimismo, las principales dificultades que tienen los médicos residentes para culminar su proyecto son el no tener la asesoría de docentes que realicen publicaciones científicas de manera regular, y el no haberse graduado de bachilleres de medicina con tesis en la facultad de medicina donde realizó estudios de pregrado ${ }^{(16)}$. En cambio, los médicos residentes de programas extranjeros logran culminar con mayor frecuencia proyectos de investigación, e incluso publicarlos en el transcurso del RM ${ }^{(17,18)}$.

Respecto a la visibilidad de las publicaciones, la totalidad de los artículos fueron realizados en idioma español. Si bien es cierto que las revistas biomédicas que tienen mayor impacto y visibilidad están publicadas en idioma inglés, debemos considerar que la mayoría de los resultados de los proyectos generados por los médicos residentes son de interés local o regional, por lo que publicarlo en idioma español facilita su difusión ${ }^{(19,20)}$. Sobre las bases de indización, solo una minoría publicó en revistas indizadas en Medline, la mayoría de veces logrado por egresados de la especialidad de gastroenterología, esto puede deberse a que la Revista Peruana de Gastroenterología, está indizada a Medline.

Como limitaciones del estudio se considera el haber utilizado un esquema de búsqueda de publicaciones que no ha sido validado, sin embargo este esquema ha sido utilizado en publicaciones anteriores ${ }^{(7-9,16)}$. Tampoco se ha considerado la publicación realizado por médicos residentes en congresos u otro tipo de eventos científicos, aunque debemos considerar que estos generalmente son informes preliminares y no son considerados en las mediciones bibliométricas. También hay que considerar que no todas las revistas de las sociedades científicas peruanas se encuentran actualizadas. 
En conclusión, el 23,6\% de los proyectos de investigación generados por médicos residentes de la Facultad de Medicina de la Universidad Peruana Cayetano Heredia para su titulación como especialistas logra ser publicado en una revista científica. Recomendamos que para incrementar dicha frecuencia se debe seguir usando el formato de publicación en revista científica, además de diseñar estrategias que estimulen esta actividad.
Contribuciones de autoría: RT y PY han participado en la concepción del artículo, la recolección de datos, el análisis de datos, su redacción y aprobación de la versión final. FS brindó la información y participó en la aprobación de la versión final.

Fuentes de financiamiento: autofinanciado.

Conflictos de interés: los autores declaran no tener conflictos de interés.

\section{REFERENCIAS BIBLIOGRÁFICAS}

1. United Nations Educational, Scientific and Cultural Organization (UNESCO). UNESCO science report 2010. The Current Status of Science around the World. Paris: UNESCO; 2010.

2. Pérez-Rancel M. Aspectos psicosociales de la actividad científica en la facultad de medicina. RFM. 2000;23(1):28-33.

3. Perú. Decreto Supremo No 008-88SA. Normas básicas del Sistema Nacional de Residentado Médico. Lima, 18 de Febrero de 1988.

4. Perú. Resolución Suprema No 002 2006-SA. Reglamento del Sistema Nacional de Residentado Médico. Lima, 01 de marzo del 2006

5. Concha M, Christian Miranda J, Vergara $\mathrm{G}$, Ibarra $\mathrm{H}$. Intención y desarrollo de competencias en investigación clínica en programas de postítulo de médico especialista en Chile. Rev Med Chile. 2012;140:326-33.

6. Raiher S. Estudio comparativo entre la producción científica latinoamericana y mundial en inequidades en salud durante los años 1999-2008. Tesis para el grado de Licenciada en Bibliotecología y Documentación. La Plata, Argentina: Universidad Nacional de La Plata; 2008.

7. Arriola-Quiroz I, Walter H. Curioso, Cruz-Encarnación M, Gayoso O. Characteristics and publication patterns of theses from a Peruvian medical school. Health Inf Libr J. 2010;27(2):148-54.

8. Vine R. Google Scholar.J Med Libr Assoc. 2006;94(1):97-9.
9. Curioso WH, Arriola-Quiroz I, Cruz-Encarnación M. A simple strategy to improve searching of indexed articles in SciELO. Rev Med Chil. 2008 Jun;136(6):812-4. doi: /S003498872008000600020 .

10. Gill S, Levin A, Djurdjev O, Yoshida EM. Obstacles to residents' conducting research and predictors of publication. Acad Med. 2001;76(5):477.

11. Gutiérrez C, Mayta P. Publicación desde el pregrado en Latinoamérica. Importancia, limitaciones y alternativas de solución. CIMEL. 2003;8(1):54-60.

12. Johnson SH. Adapting a thesis to publication style: meeting editors' expectations. Dimens Crit Care Nurs. 1996 May-Jun;15(3):160-7.

13. Mills LS, Steiner AZ, Rodman AM, Donnell CL, Steiner MJ. Trainee participation in an annual research day is associated with future publications. Teach Learn Med. 2011 Jan;23(1):62-7. doi: 10.1080/10401334.2011.536895.

14. Kurahara DK, Kogachi K, Yamane M, Ly CL, Foster JH, Masaki-Tesoro $\mathrm{T}$, et al. A pediatric residency research requirement to improve collaborative resident and faculty publication productivity. Hawaii J Med Public Health. 2012 Aug;71(8):224-8.

15. Shakiba B, IraniS, Salmasian H. A jumpstart for student researchers in Iran. Lancet. 2007 Apr 7;369(9568):1167-8.

16. Ticse R, Pamo O, Samalvides F, Quispe T. Factores asociados a la culminación del proyecto de investigación requerido para optar el título de especialista en una universidad peruana. Rev Peru Med Exp Salud Publica. 2014;31(1):48-55.

17. Yang G, Zaid UB, Erickson BA, Blaschko SD, Carroll PR, Breyer BN. Urology resident publication output and its relationship to future academic achievement. J Urol. 2011 Feb;185(2):642-6. doi: 10.1016/j.juro.2010.09.097.

18. Manjarin M, Cutri AM, Torres FA, Noguerol ME, Ossorio MF, Durán $\mathrm{P}$, et al. Evaluación de la producción científica en la residencia de pediatría. Arch Argent Pediatr. 2009 Feb;107(1):26-9. doi: 10.1590/S032500752009000100007.

19. Cardinali DP. Posibles estrategias para la promoción de publicaciones científicas regionales. En: Impacto y visibilidad de las revistas científicas. Buenos Aires: Biblioteca Nacional; 2011. p 63-6.

20. Huamaní C, Chávez-Solís P, Domínguez-Haro W, Solano-Aldana M. Producción científica estudiantil: análisis y expectativas. Rev Peru Med Exp Salud Publica. 2007;24(4):444-6.

Correspondencia: Ray Ticse Aguirre

Dirección: Servicio de Endocrinología. Hospital Nacional Cayetano Heredia. Av. Honorio Delgado 262. San Martin de Porres. Teléfono:4820402

Correo electrónico: ray.ticse@upch.pe 